\title{
CONVERGENCE RATES IN A WEIGHTED FUC̆IK PROBLEM
}

\author{
ARIEL M. SALORT
}

\begin{abstract}
In this work we consider the Fučik problem for a family of weights depending on $\varepsilon$ with Dirichlet and Neumann boundary conditions. We study the homogenization of the spectrum. We also deal with the special case of periodic homogenization and we obtain the rate of convergence of the first non-trivial curve of the spectrum.
\end{abstract}

\section{INTRODUCTION}

Given a bounded domain $\Omega$ in $\mathbb{R}^{N}, N \geq 1$ we study the asymptotic behavior as $\varepsilon \rightarrow 0$ of the spectrum of the following asymmetric elliptic problem

$$
-\Delta_{p} u_{\varepsilon}=\alpha_{\varepsilon} m_{\varepsilon}\left(u_{\varepsilon}^{+}\right)^{p-1}-\beta_{\varepsilon} n_{\varepsilon}\left(u_{\varepsilon}^{-}\right)^{p-1} \quad \text { in } \Omega
$$

either with homogeneous Dirichlet or Neumann boundary conditions.

Here, $\Delta_{p} u=\operatorname{div}\left(|\nabla u|^{p-2} \nabla u\right)$ is the $p$-Laplacian with $1<p<\infty$ and $u^{ \pm}:=$ $\max \{ \pm u, 0\}$. The parameters $\alpha_{\varepsilon}$ and $\beta_{\varepsilon}$ are reals and depending on $\varepsilon>0$. We assume that the family of weight functions $m_{\varepsilon}$ and $n_{\varepsilon}$ are positive and uniformly bounded away from zero.

For a moment let us focus problem (1.1) for fixed $\varepsilon>0$ with positive weights $m(x), n(x)$ :

$$
-\Delta_{p} u=\alpha m(x)\left(u^{+}\right)^{p-1}-\beta n(x)\left(u^{-}\right)^{p-1} \quad \text { in } \Omega
$$

with Dirichlet or Neumann boundary conditions.

Consider the Fučik spectrum defined as the set

$$
\Sigma(m, n):=\left\{(\alpha, \beta) \in \mathbb{R}^{2}:(1.2) \text { has a nontrivial solution }\right\} .
$$

Let us observe that when $r=n=m$ and $\lambda=\alpha=\beta$, equation (1.2) becomes

$$
-\Delta_{p} u=\lambda r|u|^{p-2} u \quad \text { in } \Omega
$$

with Dirichlet or Neumann boundary conditions, which is the eigenvalue problem for the $p$-Laplacian. These has been widely studied. See for instance $[2,10,16,13]$ for more information.

It follows immediately that $\Sigma$ contain the lines $\lambda_{1}(m) \times \mathbb{R}$ and $\mathbb{R} \times \lambda_{1}(n)$. For this reason, we denote by $\Sigma^{*}=\Sigma^{*}(m, n)$ the set $\Sigma$ without these trivial lines. Observe that if $(\alpha, \beta) \in \Sigma^{*}$ with $\alpha \geq 0$ and $\beta \geq 0$ then $\lambda_{1}(m)<\alpha$ and $\lambda_{1}(n)<\beta$.

2010 Mathematics Subject Classification. 35B27, 35P15, 35P30.

Key words and phrases. Eigenvalue homogenization, nonlinear eigenvalues, order of convergence. 
The study of problem (1.2) with Dirichlet boundary conditions have a long history that we briefly describe below. The one-dimensional case with positive constant coefficients (i.e., $m, n \in \mathbb{R}^{+}$and $p=2$ ) was studied in the 1970s by Fučik [17] and Dancer [11] in connection with jumping nonlinearities. Properties and descriptions of the first non-trivial curve on the spectrum of (1.2) on $\mathbb{R}^{N}$ for the general case $(p \neq 2)$ without weights can be found in Cuesta, de Figueiredo and Gossez [10], Dancer and Perera [12], Drábek and Robinson [15], Perera [27].

The case with positive weights $m(x)$ and $n(x)$ was recently studied, see for instance Rynne and Walter [28], Arias and Campos [3], Drabek [14], Reichel and Walter [24]. For indefinite weights $m(x)$ and $n(x)$ see Alif and Gossez[1], Leadi and Marcos [23].

The main problem one address is to obtain a description as accurate as possible of the set $\Sigma^{*}$. In the one-dimensional case, $p=2$, without weights this description is obtained in a precise manner: the spectrum is made of a sequence of hyperbolic like curves in $\mathbb{R}^{+} \times \mathbb{R}^{+}$, see for instance [18]. When $m(x)$ and $n(x)$ are non-constants weights, in [1] it is proved a characterization of the spectrum in terms of the socalled zeroes-functions.

In $\mathbb{R}^{N}$ with $N>1$ and Dirichlet boundary conditions, only a full description of the first nontrivial curve of $\Sigma$ is known, which we will denote by $\mathcal{C}_{1}=\mathcal{C}_{1}(m, n)$.

Assuming that the weight functions $m, n$ are positive and uniformly bounded, in [4] (see Theorem 33) is proved that $\mathcal{C}_{1}$ can be characterized by

$$
\mathcal{C}_{1}=\left\{(\alpha(s), \beta(s)), s \in \mathbb{R}^{+}\right\}
$$

where $\alpha(s)$ and $\beta(s)$ are continuous functions defined by

$$
\alpha(s)=c(m, s n), \quad \beta(s)=s \alpha(s)
$$

and $c(\cdot, \cdot)$ is given by

$$
c(m, n)=\inf _{\gamma \in \Gamma} \max _{u \in \gamma(I)} \frac{A(u)}{B(u)} .
$$

where $I:=[-1,+1]$. Here, the functionals $A$ and $B$ are given by

$$
A(u)=\int_{\Omega}|\nabla u|^{p} d x, \quad B_{m, n}=\int_{\Omega} m(x)\left(u^{+}\right)^{p}+n(x)\left(u^{-}\right)^{p} d x,
$$

with

$$
\Gamma=\left\{\gamma \in C\left([-1,+1], W_{0}^{1, p}(\Omega)\right): \gamma(-1) \geq 0 \text { and } \gamma(1) \leq 0\right\} .
$$

In [4] (see Proposition 34) some important properties of the functions $\alpha(s)$ and $\beta(s)$ are proved. Namely, both $\alpha(s)$ and $\beta(s)$ are continuous, $\alpha(s)$ is strictly decreasing and $\beta(s)$ is strictly increasing. One also has that $\alpha(s) \rightarrow+\infty$ if $s \rightarrow 0$ and $\beta(s) \rightarrow+\infty$ is $s \rightarrow+\infty$.

Having defined these previous concepts and definitions, let us back to problem (1.1).

Homogenization of the spectrum of elliptic operators was extensively studied in the last years. The case of the eigenvalues of the weighted $p$-Laplacian operator in periodic settings, i.e., $-\Delta_{p} u_{\varepsilon}=\rho_{\varepsilon}\left|u_{\varepsilon}\right|^{p-2} u_{\varepsilon}$ with Dirichlet boundary conditions and $\rho_{\varepsilon}=\rho\left(\frac{x}{\varepsilon}\right), \rho$ being a $Q$-periodic function with $Q$ the unit cube in $\mathbb{R}^{N}$, together with 
a family of more general problems it was studied for instance by [8],[9],[20],[22],[26] in the linear case $(p=2)$ and by [6],[7],[21] in the non-linear case $(p \neq 2)$.

Up to our knowledge, no investigation was made in the homogenization and rates of convergence of the Fučik Spectrum. We are interested in studying the behavior as $\varepsilon \rightarrow 0$ of problem (1.1) when $m_{\varepsilon}(x)$ and $n_{\varepsilon}(x)$ are general functions depending on $\varepsilon$, and in the special case of rapidly oscillating periodic functions, i.e., $m_{\varepsilon}(x)=m(x / \varepsilon)$ and $n_{\varepsilon}(x)=n(x / \varepsilon)$ for two $Q$-periodic functions $m, n$ uniformly bounded away from zero (see assumptions (2.1)), $Q$ being the unit cube of $\mathbb{R}^{N}$.

Our main aim is to study the limit as $\varepsilon \rightarrow 0$ of the first nontrivial curve in the spectrum $\Sigma_{\varepsilon}:=\Sigma\left(m_{\varepsilon}, n_{\varepsilon}\right)$, say $\mathcal{C}_{1}^{\varepsilon}=\left\{\left(\alpha_{\varepsilon}(s), \beta_{\varepsilon}(s)\right), s \in \mathbb{R}^{+}\right\}$. We wonder: there exists a limit curve $\mathcal{C}_{1}=\left\{\left(\alpha_{0}(s), \beta_{0}(s)\right), s \in \mathbb{R}^{+}\right\}$such that

$$
\mathcal{C}_{1}^{\varepsilon} \rightarrow \mathcal{C}_{1}, \quad \text { as } \varepsilon \rightarrow 0 \text { ? }
$$

Can this limit curve be characterized like a curve of a limit problem? We will see that the answer is positive.

Therefore, a natural question arises: can the rate of convergence of $\mathcal{C}_{1}^{\varepsilon}$ be estimated? I.e., can we give an estimate of the remainders

$$
\left|\alpha_{\varepsilon}(s)-\alpha_{0}(s)\right| \text { and }\left|\beta_{\varepsilon}(s)-\beta_{0}(s)\right| \text { ? }
$$

We give positive answers to these questions in the periodic setting. In fact, in Theorem 2.6 we obtain the bounds

$$
\left|\alpha_{\varepsilon}(s)-\alpha_{0}(s)\right| \leq c(1+s) \tau(s) \varepsilon, \quad\left|\beta_{\varepsilon}(s)-\beta_{0}(s)\right| \leq c s(1+s) \tau(s) \varepsilon, \quad s \in \mathbb{R}^{+}
$$

where $c$ is a constant fully determined which is independent of $s$ and $\varepsilon$, and $\tau$ is a explicit function depending only of $s$ (see (2.7)).

Particularly, for the limit values of the coordinates, we get

$$
\left|\alpha_{\varepsilon}^{\infty}-\alpha_{0}^{\infty}\right| \leq c \varepsilon, \quad\left|\beta_{\varepsilon}^{0}-\beta_{0}^{0}\right| \leq c \varepsilon
$$

where $\alpha_{\varepsilon}^{\infty}=\lim _{s \rightarrow \infty} \alpha_{\varepsilon}(s), \alpha_{0}^{\infty}=\lim _{s \rightarrow \infty} \alpha_{0}(s), \beta_{\varepsilon}^{0}=\lim _{s \rightarrow \infty} \beta_{\varepsilon}(s), \beta_{0}^{0}=\lim _{s \rightarrow \infty} \beta_{0}(s)$ and $c$ is independent of $s$ and $\varepsilon$.

\section{The RESUlts}

Let $\Omega \subset \mathbb{R}^{N}$ be a bounded domain and $\varepsilon$ a real positive number. We consider functions $m_{\varepsilon}, n_{\varepsilon}$ such that for constants $m_{-} \leq m_{+}, n_{-} \leq n_{+}$

$$
0<m_{-} \leq m_{\varepsilon}(x) \leq m_{+} \leq+\infty \quad \text { and } \quad 0<n_{-} \leq n_{\varepsilon}(x) \leq n_{+} \leq+\infty .
$$

Also, we assume that there exist functions $m_{0}(x)$ and $n_{0}(x)$ satisfying (2.1) such that, as $\varepsilon \rightarrow 0$,

$$
\begin{array}{ll}
m_{\varepsilon}(x) \rightarrow m_{0}(x) & \text { weakly* in } L^{\infty}(\Omega) \\
n_{\varepsilon}(x) \rightarrow n_{0}(x) & \text { weakly* in } L^{\infty}(\Omega) .
\end{array}
$$

First, we address the problem with Dirichlet boundary conditions.

When $\varepsilon \rightarrow 0$ the natural limit problem for (1.1) is the following

$$
\begin{cases}-\Delta_{p} u_{0}=\alpha_{0} m_{0}(x)\left(u_{0}^{+}\right)^{p-1}-\beta_{0} n_{0}(x)\left(u_{0}^{-}\right)^{p-1} & \text { in } \Omega \\ u_{0}=0 & \text { on } \partial \Omega\end{cases}
$$

where $m_{0}$ and $n_{0}$ are given in (2.2). 
The main result is the following:

Theorem 2.1. Let $m_{\varepsilon}, n_{\varepsilon}$ satisfying (2.1) and (2.2). Then the first non-trivial curve of problem (1.1)

$$
\mathcal{C}_{\varepsilon}:=\mathcal{C}_{1}\left(m_{\varepsilon}, n_{\varepsilon}\right)=\left\{\alpha_{\varepsilon}(s), \beta_{\varepsilon}(s), s \in \mathbb{R}^{+}\right\}
$$

converges to the first non-trivial curve of the limit problem (2.3)

$$
\mathcal{C}:=\mathcal{C}_{1}\left(m_{0}, n_{0}\right)=\left\{\alpha_{0}(s), \beta_{0}(s), s \in \mathbb{R}^{+}\right\}
$$

as $\varepsilon \rightarrow 0$ in the sense that $\alpha_{\varepsilon}(s) \rightarrow \alpha_{0}(s)$ and $\beta_{\varepsilon}(s) \rightarrow \beta_{0}(s) \forall s \in \mathbb{R}^{+}$.

Remark 2.2. Let us consider the weighted $p$-Laplacian problem

$$
\begin{cases}-\Delta_{p} u=\lambda r_{\varepsilon}(x)|u|^{p-2} u & \text { in } \Omega \\ u=0 & \text { on } \partial \Omega\end{cases}
$$

where $r_{\varepsilon}$ is a function such that $r_{\varepsilon}(x) \rightarrow r(x)$ weakly* in $L^{\infty}(\Omega)$ as $\varepsilon$ tends to zero. It is well-known that the first eigenvalue of (2.4) converges to the first eigenvalue of the $p$-Laplacian equation with weight $r(x)$, see for instance [6]. The fact that the trivial lines of $\Sigma_{\varepsilon}$ are defined by $\lambda_{1}\left(m_{\varepsilon}\right) \times \mathbb{R}$ and $\mathbb{R} \times \lambda_{1}\left(n_{\varepsilon}\right)$ it allows us to affirm the convergence of the trivial lines to those of the limit problem.

Remark 2.3. Using the variational characterization of the second (variational) eigenvalue of [4], Theorem 2.1 implies the convergence of the second (variational) eigenvalue of (2.4) to those of the limit problem, which recover result recently proved in [21] for the case of the weighted $p$-Laplacian. However, the results in [21] consider a more general class of quasilenar operators and $\varepsilon$-dependence on the operator as well.

In the important case of periodic homogenization, i.e., when $m_{\varepsilon}(x)=m(x / \varepsilon)$ and $n_{\varepsilon}(x)=n(x / \varepsilon)$ where $m$ and $n$ are $Q$-periodic functions, $Q$ being the unit cube in $\mathbb{R}^{N}$, we have that $m_{0}=\bar{m}$ and $n_{0}=\bar{n}$ are real numbers given by the averages of $m$ and $n$ over $Q$, respectively. Consequently, the limit problem (2.3) becomes

$$
\begin{cases}-\Delta_{p} u_{0}=\alpha_{0} \bar{m}\left(u_{0}^{+}\right)^{p-1}-\beta_{0} \bar{n}\left(u_{0}^{-}\right)^{p-1} & \text { in } \Omega \\ u_{0}=0 & \text { on } \partial \Omega .\end{cases}
$$

In this case, besides the convergence of the curves given in Theorem 2.1 and Remark 2.2 , we obtain the convergence rates.

First, by using the variational characterization of the first eigenvalue of (2.4) we analyze the trivial lines of $\Sigma_{\varepsilon}$ :

Theorem 2.4. Let $m_{\varepsilon}, n_{\varepsilon}$ weights satisfying (2.1) and (2.2) given in terms of $Q$-periodic functions $m, n$ in the form $m_{\varepsilon}(x)=m\left(\frac{x}{\varepsilon}\right)$ and $n_{\varepsilon}(x)=n\left(\frac{x}{\varepsilon}\right)$. Let us denote by $\lambda_{1}\left(m_{\varepsilon}\right), \lambda_{1}\left(n_{\varepsilon}\right), \lambda_{1}(\bar{m})$ and $\lambda_{1}(\bar{n})$ to the first eigenvalue of equation (2.4) with weight $m_{\varepsilon}, n_{\varepsilon}, \bar{m}$ and $\bar{n}$, respectively. Then

$$
\left|\lambda_{1}\left(m_{\varepsilon}\right)-\lambda_{1}(\bar{m})\right| \leq C_{m} \varepsilon, \quad\left|\lambda_{1}\left(n_{\varepsilon}\right)-\lambda_{1}(\bar{n})\right| \leq C_{n} \varepsilon,
$$

with $C_{m}$ given by

$$
C_{m}=p c_{1}\|m-\bar{m}\|_{L^{\infty}\left(\mathbb{R}^{N}\right)}\left(m_{+}\right)^{1 / p}\left(m_{-}\right)^{-\frac{1}{p}-2} \mu_{1}^{\frac{1}{p}+1}
$$

where $\mu_{1}$ is the first eigenvalue of the Dirichlet $p$-Laplacian and $c_{1} \leq \sqrt{N} / 2$. 
Remark 2.5. From Theorem 2.4 it follows the convergence rates of the trivial lines of $\Sigma_{\varepsilon}$ : if $p_{\varepsilon} \in \lambda_{1}\left(m_{\varepsilon}\right) \times \mathbb{R}$, we get

$$
\left|p_{\varepsilon}-p_{0}\right| \leq C_{m} \varepsilon
$$

where $p_{0}$ belongs to the line $\lambda_{1}(\bar{m}) \times \mathbb{R}$. Analogously for $p_{\varepsilon} \in \mathbb{R} \times \lambda_{1}\left(n_{\varepsilon}\right)$.

Related to the first nontrivial curve of $\Sigma_{\varepsilon}$ we obtain:

Theorem 2.6. Under the same considerations of Theorem 2.1, if the weights $m_{\varepsilon}$ and $n_{\varepsilon}$ are given in terms of $Q$-periodic functions $m, n$ in the form $m_{\varepsilon}(x)=m\left(\frac{x}{\varepsilon}\right)$ and $n_{\varepsilon}(x)=n\left(\frac{x}{\varepsilon}\right)$, for each $s \in \mathbb{R}^{+}$, we have the following estimates

$$
\left|\alpha_{\varepsilon}(s)-\alpha_{0}(s)\right| \leq c(1+s) \tau(s) \varepsilon, \quad\left|\beta_{\varepsilon}(s)-\beta_{0}(s)\right| \leq c s(1+s) \tau(s) \varepsilon
$$

where $c$ is given explicitly by

$$
p c_{1} c_{p}^{p-1} \max \left\{\|m-\bar{m}\|_{L^{\infty}\left(\mathbb{R}^{N}\right)},\|n-\bar{n}\|_{L^{\infty}\left(\mathbb{R}^{N}\right)}\right\}\left(\min \left\{m_{-}^{-1}, n_{-}^{-1}\right\} \mu_{2}\right)^{2}
$$

where $c_{1}$ and $c_{p}$ are the Poincarés constant in $L^{1}(Q)$ and $L^{p}(\Omega)$, respectively, $\mu_{2}$ is the second Dirichlet $p$-Laplacian eigenvalue in $\Omega$ and $\tau$ is defined by

$$
\tau(s)= \begin{cases}1 & s \geq 1 \\ s^{-2} & s<1 .\end{cases}
$$

Remark 2.7. According to Proposition 34 and Proposition 35 in [4], when $p \leq N$ the limits of $\alpha_{\varepsilon}(s), \alpha_{0}(s)$ as $s \rightarrow \infty$ and $\beta_{\varepsilon}(s), \beta_{0}(s)$ as $s \rightarrow 0$ can be characterized in terms of the first eigenvalues of weighted $p$-Laplacian problems. Moreover, $\lim _{s \rightarrow \infty} \alpha_{\varepsilon}(s)=\lambda_{1}\left(m_{\varepsilon}\right)$ and $\lim _{s \rightarrow 0} \beta_{\varepsilon}(s)=\lambda_{1}\left(n_{\varepsilon}\right)$. Similarly for $\alpha_{0}$ and $\beta_{0}$. Consequently, by using the estimates obtained in Theorem 2.4 , it is easy to compute the convergence rates in the limit cases when the periodic case is considered, namely

$$
\begin{aligned}
& \lim _{s \rightarrow \infty}\left|\alpha_{\varepsilon}(s)-\alpha_{0}(s)\right|=\left|\lambda_{1}\left(m_{\varepsilon}\right)-\lambda_{1}(\bar{m})\right| \leq C_{m} \varepsilon, \\
& \lim _{s \rightarrow 0}\left|\beta_{\varepsilon}(s)-\beta_{0}(s)\right|=\left|\lambda_{1}\left(n_{\varepsilon}\right)-\lambda_{1}(\bar{n})\right| \leq C_{n} \varepsilon .
\end{aligned}
$$

Now we focus our attention on the Neumann boundary conditions case, i.e., we study the homogenization of the spectrum of the Fučik problem

$$
\begin{cases}-\Delta_{p} u=\alpha m(x)\left(u^{+}\right)^{p-1}-\beta n(x)\left(u^{-}\right)^{p-1} & \text { in } \Omega \\ \frac{\partial u}{\partial \eta}=0 & \text { on } \partial \Omega .\end{cases}
$$

where $\partial u / \partial \eta=\nabla u \cdot \eta$ denotes the unit exterior normal. In Section 4 we study the limit problem associated to (2.8) and the homogenization of the first non-trivial curve of its spectrum. We obtain similar results to the Dirichlet case: in Theorem 4.1 we study the convergence in general settings; in Theorem 4.2 we deal with the periodic case, obtaining convergence rates similar to those of Theorem 2.6.

\section{Proof of the Dirichlet Results}

We begin with the proof of the Theorem 2.4. For that, we will use a technical result proved in [21] that is essential to estimate the rate convergence of the eigenvalues since allows us to replace an integral involving a rapidly oscillating function with one that involves its average in the unit cube. 
Theorem 3.1 (Theorem 3.4 in [21].). Let $\Omega$ be a bounded domain in $\mathbb{R}^{N}, N \geq 1$. Let $g \in L^{\infty}\left(\mathbb{R}^{N}\right)$ be a $Q$-periodic function, being $Q=[0,1]^{N}$ the unit cube in $\mathbb{R}^{N}$, such that $0<g^{-} \leq g \leq g^{+}<+\infty$ for $g^{ \pm}$constants. Then

$$
\left.\left|\int_{\Omega}\left(g\left(\frac{x}{\varepsilon}\right)-\bar{g}\right)\right| u\right|^{p} \mid \leq p c_{1}\|g-\bar{g}\|_{L^{\infty}\left(\mathbb{R}^{N}\right)} \varepsilon\|u\|_{L^{p}(\Omega)}^{p-1}\|\nabla u\|_{L^{p}(\Omega)}
$$

for every $u \in W_{0}^{1, p}(\Omega)$ where $1<p<+\infty, \Omega \subset \mathbb{R}^{N}$ bounded domain and $\bar{g}:=\int_{Q} g$. Here, $c_{1}$ is the optimal constant in Poincaré's inequality in $L^{1}(Q)$ which satisfies $c_{1} \leq \sqrt{N} / 2$.

Remark 3.2. Sometimes it will be useful to use an inequality involving only the gradient. By using Poincaré's inequality we can bound $\|u\|_{L^{p}(\Omega)}^{p-1} \leq c_{p}(\Omega)^{p-1}\|\nabla u\|_{L^{p}(\Omega)}^{p-1}$. With the same assumptions of Theorem 3.1, it allow us to rewrite inequality (3.1) as

$$
\left.\left|\int_{\Omega}\left(g\left(\frac{x}{\varepsilon}\right)-\bar{g}\right)\right| u\right|^{p} \mid \leq C \varepsilon\|\nabla u\|_{L^{p}(\Omega)}^{p},
$$

where $C=p c_{1} c_{p}^{p-1}\|g-\bar{g}\|_{L^{\infty}\left(\mathbb{R}^{N}\right)}$.

Proof of Theorem 2.4: $\lambda_{1}(\bar{m})$ can be characterized variationally as

$$
\lambda_{1}(\bar{m})=\inf _{u \in W_{0}^{1, p}(\Omega)} \frac{\int_{\Omega}|\nabla u|^{p}}{\int_{\Omega} \bar{m}|u|^{p}}=\frac{\int_{\Omega}\left|\nabla u_{1}\right|^{p}}{\int_{\Omega} \bar{m}\left|u_{1}\right|^{p}}+o(1)
$$

for some $u_{1} \in W_{0}^{1, p}(\Omega)$. We can bound

$$
\lambda_{1}\left(m_{\varepsilon}\right)=\inf _{u \in W_{0}^{1, p}(\Omega)} \frac{\int_{\Omega}|\nabla u|^{p}}{\int_{\Omega} m_{\varepsilon}|u|^{p}} \leq \frac{\int_{\Omega}\left|\nabla u_{1}\right|^{p}}{\int_{\Omega} \bar{m}\left|u_{1}\right|^{p}} \frac{\int_{\Omega} \bar{m}\left|u_{1}\right|^{p}}{\int_{\Omega} m_{\varepsilon}\left|u_{1}\right|^{p}} .
$$

By using Theorem 3.1, (2.1) and (3.2) it follows that

$$
\begin{aligned}
\frac{\int_{\Omega} \bar{m}\left|u_{1}\right|^{p}}{\int_{\Omega} m_{\varepsilon}\left|u_{1}\right|^{p}} & \leq 1+c \varepsilon \frac{\left(\int_{\Omega}\left|u_{1}^{p}\right|\right)^{\frac{p-1}{p}}\left(\int_{\Omega}\left|\nabla u_{1}\right|^{p}\right)^{\frac{1}{p}}}{\int_{\Omega} m_{\varepsilon}\left|u_{1}\right|^{p}} \\
& \leq 1+c \varepsilon \frac{\bar{m}^{1 / p}}{m_{-}} \frac{\left(\int_{\Omega}\left|u_{1}^{p}\right|\right)^{\frac{p-1}{p}}\left(\int_{\Omega}\left|\nabla u_{1}\right|^{p}\right)^{\frac{1}{p}}}{\int_{\Omega} \bar{m}\left|u_{1}\right|^{p}} \\
& \leq 1+C \varepsilon\left(\frac{\int_{\Omega}\left|\nabla u_{1}\right|^{p}}{\int_{\Omega} \bar{m}\left|u_{1}\right|^{p}}\right)^{\frac{1}{p}} \leq 1+C \varepsilon\left(\lambda_{1}(\bar{m})+o(1)\right)^{\frac{1}{p}},
\end{aligned}
$$

where $C=p c_{1}\|m-\bar{m}\|_{L^{\infty}\left(\mathbb{R}^{N}\right)}\left(m_{+}\right)^{1 / p} / m_{-}$.

By replacing (3.4) and (3.2), in (3.3) we get

$$
\lambda_{1}\left(m_{\varepsilon}\right)-\lambda_{1}(\bar{m}) \leq C \varepsilon \lambda_{1}(\bar{m})^{\frac{1}{p}+1} .
$$

In a similar way, interchanging the roles of $\lambda_{1}\left(m_{\varepsilon}\right)$ and $\lambda_{1}(\bar{m})$ we obtain

$$
\lambda_{1}(\bar{m})-\lambda_{1}\left(m_{\varepsilon}\right) \leq C \varepsilon \lambda_{1}\left(m_{\varepsilon}\right)^{\frac{1}{p}+1} .
$$

By using (2.1) immediately it follows that

$$
\max \left\{\lambda_{1}(\bar{m}), \lambda_{1}\left(m_{\varepsilon}\right)\right\} \leq\left(m_{-}\right)^{-1} \mu_{1},
$$

where $\mu_{1}$ is the first eigenvalue of the Dirichlet $p$-Laplacian.

From equations (3.5), (3.6) and (3.7) it follows the result. 
In the next Lemma we obtain upper bounds for the coordinates of the first curve of $\Sigma^{*}(m, n)$.

Lemma 3.3. Let $m, n$ satisfying (2.1) and let $(\alpha(s), \beta(s)) \in \mathcal{C}_{1}(m, n)$. Then for each $s \in \mathbb{R}^{+}$,

$$
\alpha(s) \leq \min \left\{m_{-}^{-1}, n_{-}^{-1}\right\} \mu_{2} \tau(s), \quad \beta(s) \leq \min \left\{m_{-}^{-1}, n_{-}^{-1}\right\} \mu_{2} s \tau(s)
$$

with $\tau$ defined by

$$
\tau(s)= \begin{cases}1 & s \geq 1 \\ s^{-1} & s \leq 1 .\end{cases}
$$

where $m_{-}, n_{-}$are given by (2.1) and $\mu_{2}$ is the second eigenvalue of the $p$-Laplacian equation in $\Omega$ without weights and Dirichlet boundary conditions.

Proof. Let $s \in \mathbb{R}^{+}$. When the parameter $s \geq 1$ we can bound

$$
\lambda_{1}(m) \leq \alpha(s) \leq \alpha(1)=c(m, n) .
$$

Let $\lambda_{2}(m)$ be the second eigenvalue of the problem (1.3) with weight $m(x)$. It satisfies that $\alpha(1) \leq \min \left\{\lambda_{2}(m), \lambda_{2}(n)\right\}$. By using the assumptions (2.1) over $m(x)$, we can bound $\lambda_{2}(m)$ by $\mu_{2} m_{-}^{-1}$, where $\mu_{2}$ is the second eigenvalue of the $p$-Laplacian equation with Dirichlet boundary conditions on $\Omega$. Analogously for $\lambda_{2}(n)$. We get

$$
\alpha(s) \leq \alpha(1) \leq \min \left\{m_{-}^{-1}, n_{-}^{-1}\right\} \mu_{2}, \quad s \geq 1
$$

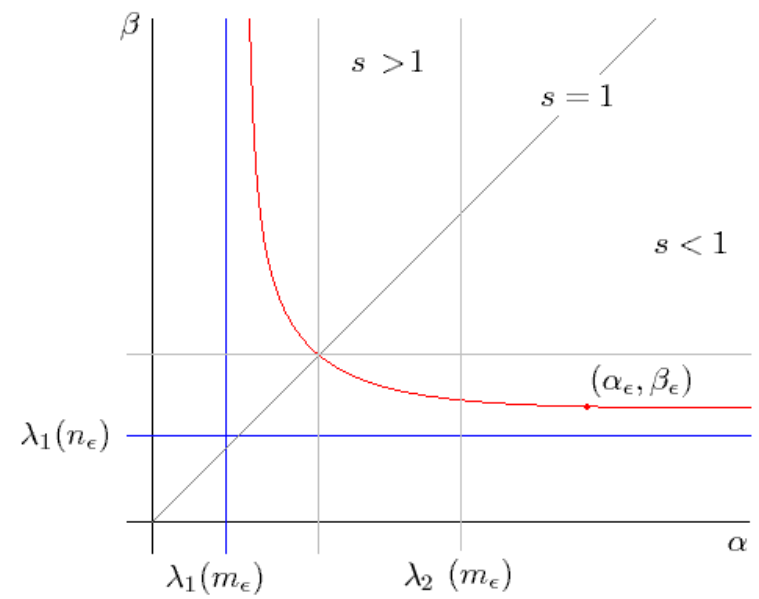

Figure 1. The first curve of the spectrum.

When $s \leq 1$ the following bound holds for the second coordinate of $\mathcal{C}_{\varepsilon}$

$$
\lambda_{1}(n) \leq \beta(s) \leq \beta(1) .
$$

By multiplying (3.10) by $s^{-1}$ and by using that $\beta(s)=s \alpha(s)$ we have

$$
s^{-1} \lambda_{1}(n) \leq \alpha(s) \leq s^{-1} \beta(1) .
$$


Being $\alpha(1)=\beta(1)$, it follows that

$$
\alpha(s) \leq s^{-1} \alpha(1) \leq s^{-1} \min \left\{m_{-}^{-1}, n_{-}^{-1}\right\} \mu_{2}, \quad s \leq 1 .
$$

By using (3.9), (3.11) and the relation $\beta(s)=s \alpha(s)$ the conclusions of the lemma follows.

The following Proposition gives the monotonicity of $c(\cdot, \cdot)$ :

Proposition 3.4 (Proposition 23, [4]). If $m \leq \tilde{m}$ and $n \leq \tilde{n}$ a.e., then

$$
c(\tilde{m}, \tilde{n}) \leq c(m, n),
$$

where $c(\cdot, \cdot)$ is defined by $(1.6)$.

In the next Lemma we obtain lower bounds for the coordinates of the first curve of $\Sigma^{*}(m, n)$.

Lemma 3.5. Let $m, n$ satisfying (2.1) and let $(\alpha(s), \beta(s)) \in \mathcal{C}(m, n)$. Then for each $s \in \mathbb{R}^{+}$,

with $\omega$ defined by

$$
\alpha(s) \geq \frac{1}{s} C \omega(s), \quad \beta(s) \geq C \omega(s)
$$

$$
\omega(s)= \begin{cases}1 & s \geq 1 \\ s & s \leq 1\end{cases}
$$

where $C$ is a positive constant depending only of the bounds given in (2.1).

Proof. Let $s \in \mathbb{R}^{+}$. When the parameter $s \geq 1$ we can bound bellow

$$
\beta(s) \geq \beta(1)=c(m, n), \quad s \geq 1 .
$$

Using the relation $\beta(s)=s \alpha(s)$ we obtain

$$
\alpha(s) \geq s^{-1} c(m, n), \quad s \geq 1 .
$$

Similarly, when $s \leq 1$ we have

$$
\alpha(s) \geq \alpha(1)=c(m, n), \quad s \leq 1,
$$

and again, by the relation between $\alpha(s)$ and $\beta(s)$ we get

$$
\beta(s) \geq s c(m, n), \quad s \leq 1,
$$

Using (2.1) and Proposition 3.4, we can bound bellow

$$
c(m, n) \geq c\left(m_{+}, n_{+}\right) .
$$

and the result follows.

Now we are able to prove Theorem 2.6.

Proof of Theorem 2.6: For each fixed value of $\varepsilon>0$, by (2.1) together with the monotonicity of $c(\cdot, \cdot)$ provided by Proposition 3.4, we can assert that there exist two curves $\mathcal{C}_{1}^{+}\left(m_{+}, n_{+}\right)$and $\mathcal{C}_{1}^{-}\left(m_{-}, n_{-}\right)$such that delimit above and below to the curve $\mathcal{C}_{1}^{\varepsilon}\left(m_{\varepsilon}, n_{\varepsilon}\right)$. It follows that for each fixed value of $s, \alpha_{\varepsilon}(s)$ and $\beta_{\varepsilon}(s)$ are bounded. 
Let $\left(\alpha_{\varepsilon}, \beta_{\varepsilon}\right)$ be a point belonging to the curve $\mathcal{C}_{1}^{\varepsilon}\left(m_{\varepsilon}, n_{\varepsilon}\right)$ and let $\left(\alpha_{0}, \beta_{0}\right)$ be the point obtained when $\varepsilon \rightarrow 0$. Let us see that it belongs to $\mathcal{C}_{1}(\bar{m}, \bar{n})$.

Fixed a value of $\varepsilon>0$ and by using (1.6), the inverse of $c\left(m_{\varepsilon}, n_{\varepsilon}\right)$ can be written as

$$
\frac{1}{c\left(m_{\varepsilon}, n_{\varepsilon}\right)}=\sup _{\gamma \in \Gamma} \inf _{u \in \gamma[-1,+1]} B_{m_{\varepsilon}, n_{\varepsilon}}(u)
$$

where

$$
\Gamma=\{\gamma \in C(I, H): \gamma(-1) \geq 0 \text { and } \gamma(1) \leq 0\}
$$

for $I:=[-1,+1]$ and

$$
H=\left\{u \in W_{0}^{1, p}(\Omega): A(u)=1\right\}
$$

$A$ and $B$ being the functionals defined in (1.7).

By (1.5) and (3.13) we have the following characterization for the inverse of $\alpha_{\varepsilon}(s)$

$$
\frac{1}{\alpha_{\varepsilon}(s)}=\frac{1}{c\left(m_{\varepsilon}, s n_{\varepsilon}\right)}=\sup _{\gamma \in \Gamma} \inf _{u \in \gamma(I)} B_{m_{\varepsilon}, s n_{\varepsilon}}(u) .
$$

Similarly, we can consider an equation analog to (3.14) for the representation of the inverse of $\alpha_{0}(s)$. Let $\delta>0$ and $\gamma_{1}(\delta) \in \Gamma$ such that

$$
\frac{1}{\alpha_{0}(s)}=\inf _{u \in \gamma_{1}(I)} B_{\bar{m}, s \bar{n}}(u)+O(\delta) \text {. }
$$

In order to find a bound for $a_{\varepsilon}$ we use $\gamma_{1} \in \Gamma_{1}$, which is admissible in its variational characterization,

$$
\frac{1}{\alpha_{\varepsilon}(s)} \geq \inf _{u \in \gamma_{1}(I)} B_{m_{\varepsilon}, s n_{\varepsilon}}(u)
$$

As $u \in W_{0}^{1, p}(\Omega)$, it follows that $\left(u^{+}\right)^{p}$ and $\left(u^{-}\right)^{p}$ belong to $W_{0}^{1,1}(\Omega)$. This allows us to estimate the error by replacing the oscillating weights by their averages by using Remark 3.2. For each fixed function $u \in \gamma_{1}(I)$ we bound

$$
B_{m_{\varepsilon}, s n_{\varepsilon}}(u) \geq B_{\bar{m}, s \bar{n}}(u)-c_{m} \varepsilon\left\|\nabla u^{+}\right\|_{L^{p}(\Omega)}^{p}-c_{n} \varepsilon s\left\|\nabla u^{-}\right\|_{L^{p}(\Omega)}^{p}
$$

where $c_{m}$ and $c_{m}$ are the constants given in Remark 3.2. As $u \in H$ we have

$$
\left\|\nabla u^{+}\right\|_{L^{p}(\Omega)}^{p} \leq 1, \quad\left\|\nabla u^{-}\right\|_{L^{p}(\Omega)}^{p} \leq 1 .
$$

So, from (3.18) and (3.17), taking $c=\max \left\{c_{m}, c_{n}\right\}$ we get

$$
B_{m_{\varepsilon}, s n_{\varepsilon}}(u) \geq B_{\bar{m}, s \bar{n}}(u)-c \varepsilon(1+s) .
$$

Taking infimum over the functions $u$ in $\gamma_{1}(I)$ together with (3.15) and (3.16) we obtain

$$
\alpha_{\varepsilon}^{-1}(s)-\alpha_{0}^{-1}(s) \geq-c \varepsilon(1+s)+O(\delta) .
$$

Letting $\delta \rightarrow 0$ we get

$$
\alpha_{\varepsilon}^{-1}(s)-\alpha_{0}^{-1}(s) \geq-c \varepsilon(1+s) .
$$

In a similar way, interchanging the roles of $\alpha_{\varepsilon}$ and $\alpha_{0}$ we obtain the inequality

$$
\alpha_{\varepsilon}^{-1}(s)-\alpha_{0}^{-1}(s) \leq c \varepsilon(1+s) .
$$

From equations (3.20) and (3.21) it follows that

$$
\left|\alpha_{\varepsilon}(s)-\alpha_{0}(s)\right| \leq c \varepsilon(1+s) \alpha_{\varepsilon}(s) \alpha_{0}(s) .
$$


By using Lemma 3.3 we can bound the expression (3.22) as

$$
\left|\alpha_{\varepsilon}(s)-\alpha_{0}(s)\right| \leq c\left(\min \left\{m_{-}^{-1}, n_{-}^{-1}\right\} \mu_{2}\right)^{2}(1+s) \tau(s)^{2} \varepsilon .
$$

where $\tau(s)$ is given by (3.8) and $\mu_{2}$ is the second eigenvalue of the Dirichlet $p$-Laplacian.

From the convergence of $\alpha_{\varepsilon}$ together with (1.5) it follows the convergence of $\beta_{\varepsilon}$ and of the whole curve.

The proof of Theorem 2.1, where general weights are considered, is analogous to that of Theorem 2.6 but we need a result similar to Theorem 3.1 that works without assuming periodicity. It is found in the following theorem.

Theorem 3.6. Let $\Omega \subset \mathbb{R}^{N}$ be a bounded domain. Let $g_{\varepsilon}$ be a function such that $0<g^{-} \leq g_{\varepsilon} \leq g^{+}<+\infty$ for $g^{ \pm}$constants and $g_{\varepsilon} \rightarrow g$ weakly* in $L^{\infty}(\Omega)$. Then for every $u \in W^{1, p}(\Omega)$,

$$
\lim _{\varepsilon \rightarrow 0} \int_{\Omega}\left(g_{\varepsilon}-g\right)|u|^{p}=0
$$

where $1<p<+\infty$.

Proof. The weak* convergence of $g_{\varepsilon}$ in $L^{\infty}(\Omega)$ says that $\int_{\Omega} g_{\varepsilon} \varphi \rightarrow \int_{\Omega} g \varphi$ for all $\varphi \in L^{1}(\Omega)$. Particularly, $u \in W^{1, p}(\Omega)$ implies that $|u|^{p} \in W^{1,1}(\Omega)$, it follows that $|u|^{p} \in L^{1}(\Omega)$ and the result is proved.

Proof of Theorem 2.1: The argument follows exactly as in the proof of Theorem 2.6 using the Theorem 3.6 instead of the Theorem 3.1.

\section{Neumann boundary Conditions}

Let $\Omega$ be a bounded domain in $\mathbb{R}^{N}, N \geq 1$ with Lipschitz boundary and let $m, n$ be two weights satisfying (2.1). We consider the following asymmetric elliptic problem with Neumann boundary conditions

$$
\begin{cases}-\Delta_{p} u=\alpha m(x)\left(u^{+}\right)^{p-1}-\beta n(x)\left(u^{-}\right)^{p-1} & \text { in } \Omega \\ \frac{\partial u}{\partial \eta}=0 & \text { on } \partial \Omega .\end{cases}
$$

where $\eta$ denotes the unit exterior normal.

Let $r(x)$ be a weight satisfying (2.1). Now, 0 is a principal eigenvalue of

$$
\begin{cases}-\Delta_{p} u=\lambda r(x)|u|^{p-2} u & \text { in } \Omega \\ \frac{\partial u}{\partial \nu}=0 & \text { on } \partial \Omega\end{cases}
$$

with constants as eigenfunctions. Moreover, the positivity of $r$ guaranties that 0 is the unique nonnegative principal eigenvalue, see [19].

Consequently, the Fučik spectrum $\Sigma=\Sigma(m, n)$ clearly contains the lines $\{0\} \times \mathbb{R}$ and $\mathbb{R} \times\{0\}$. We denote by $\Sigma^{*}=\Sigma^{*}(m, n)$ the set $\Sigma(m, n)$ without these two lines.

In this case, when $N>1$ only a full description of the first nontrivial curve of $\Sigma$, which we will denote by $\mathcal{C}_{1}=\mathcal{C}_{1}(m, n)$. Moreover, in [5] (see Theorem 6.1) a characterization similar to the Dirichlet case is given:

$$
\mathcal{C}_{1}=\left\{(\alpha(s), \beta(s)), s \in \mathbb{R}^{+}\right\}
$$


where $\alpha(s)$ and $\beta(s)$ are continuous functions defined by $\alpha(s)=c(m, s n), \beta(s)=$ $s \alpha(s)$ and $c(\cdot, \cdot)$ is given by

$$
c(m, n)=\inf _{\gamma \in \Gamma} \max _{u \in \gamma(J)} \frac{A(u)}{B(u)} .
$$

with $J:=[0,1]$, the functionals $A$ and $B$ given by (1.7), and

$$
\Gamma=\left\{\gamma \in C\left(J, W^{1, p}(\Omega)\right): \gamma(0) \geq 0 \text { and } \gamma(1) \leq 0\right\} .
$$

Let $m_{\varepsilon}$ and $n_{\varepsilon}$ be two functions such that satisfy (2.1) and (2.2). We consider the following problem depending on $\varepsilon>0$

$$
\begin{cases}-\Delta_{p} u_{\varepsilon}=\alpha_{\varepsilon} m_{\varepsilon}\left(u_{\varepsilon}^{+}\right)^{p-1}-\beta_{\varepsilon} n_{\varepsilon}\left(u_{\varepsilon}^{-}\right)^{p-1} & \text { in } \Omega \\ \frac{\partial u_{\varepsilon}}{\partial \nu}=0 & \text { on } \partial \Omega .\end{cases}
$$

As we made with the Dirichlet equation (1.1), we want to study the behavior of the first non-trivial curve in the spectrum of (4.5) as $\varepsilon \rightarrow 0$. When $\varepsilon$ tends to zero in (4.5), according to $(2.2)$ we obtain the following limit equation

$$
\begin{cases}-\Delta_{p} u_{0}=\alpha_{0} m_{0}(x)\left(u_{0}^{+}\right)^{p-1}-\beta_{0} n_{0}(x)\left(u_{0}^{-}\right)^{p-1} & \text { in } \Omega \\ \frac{\partial u_{0}}{\partial \nu}=0 & \text { on } \partial \Omega .\end{cases}
$$

Analogously to Theorem 2.1, we obtain the following result of convergence:

Theorem 4.1. Let $m_{\varepsilon}, n_{\varepsilon}$ satisfying (2.1), and (2.2). Then the first non-trivial curve of problem (4.5)

$$
\mathcal{C}_{1}^{\varepsilon}:=\mathcal{C}_{1}\left(m_{\varepsilon}, n_{\varepsilon}\right)=\left\{\alpha_{\varepsilon}(s), \beta_{\varepsilon}(s), s \in \mathbb{R}^{+}\right\}
$$

converges to the first non-trivial curve of the limit problem (4.6)

$$
\mathcal{C}_{1}:=\mathcal{C}_{1}\left(m_{0}, n_{0}\right)=\left\{\alpha_{0}(s), \beta_{0}(s), s \in \mathbb{R}^{+}\right\}
$$

as $\varepsilon \rightarrow 0$ in the sense that $\alpha_{\varepsilon}(s) \rightarrow \alpha_{0}(s), \beta_{\varepsilon}(s) \rightarrow \beta_{0}(s) \forall s \in \mathbb{R}^{+}$.

When the case of periodic homogenization is considered, i.e., $m_{\varepsilon}(x)=m(x / \varepsilon)$ and $n_{\varepsilon}(x)=n(x / \varepsilon)$ with $m$ and $n Q$-periodic functions, $Q$ being the unit cube in $\mathbb{R}^{N}$, the limit functions $m_{0}, n_{0}$ given in (2.2) are equal to the averages of $m$ and $n$ over $Q$, respectively, i.e., $m_{0}=\bar{m}$ and $n_{0}=\bar{n}$. Now, like in the Dirichlet case, in addition to the convergence of the first non-trivial curve, we obtain the convergence rates:

Theorem 4.2. Let $\Omega \subset \mathbb{R}^{N}, N>1$ be a bounded domain with $C^{1}$ boundary. Under the same considerations of Theorem 4.1, if the weights $m_{\varepsilon}$ and $n_{\varepsilon}$ are given in terms of $Q$-periodic functions $m, n$ in the form $m_{\varepsilon}(x)=m\left(\frac{x}{\varepsilon}\right)$ and $n_{\varepsilon}(x)=n\left(\frac{x}{\varepsilon}\right)$, for each $s \in \mathbb{R}^{+}$we have the following estimate

$$
\left|\alpha_{\varepsilon}(s)-\alpha_{0}(s)\right| \leq c(1+s) \tau(s) \varepsilon, \quad\left|\beta_{\varepsilon}(s)-\beta_{0}(s)\right| \leq c s(1+s) \tau(s) \varepsilon
$$

where $c=c(\Omega, p, m, n)$ is a constant independent of $\varepsilon$ and $s$, and $\tau$ is given by (2.7).

To prove Theorem 4.2 arguments used in the Dirichlet case fail. This is due to the fact that now the functions space is $W^{1, p}(\Omega)$ but Theorem 3.1 holds only for functions in $W_{0}^{1, p}(\Omega)$. The fact of enlarge the set of test functions is reflected in 
the need for more regularity of the domain $\Omega$. We will prove the following result which works with functions belonging to $W^{1, p}(\Omega)$.

Theorem 4.3. Let $\Omega \subset \mathbb{R}^{N}$ be a bounded domain with $C^{1}$ boundary and denote by $Q$ to the unit cube in $\mathbb{R}^{N}$. Let $g$ be a $Q$-periodic bounded function. Then for every $u \in W^{1, p}(\Omega)$ there exists a constant $c$ independent of $\varepsilon$ such that

$$
\left|\int_{\Omega}\left(g\left(\frac{x}{\varepsilon}\right)-\bar{g}\right) u\right| \leq c \varepsilon\|u\|_{W^{1, p}(\Omega)}
$$

where $\bar{g}=\int_{Q} g$ and $1 \leq p<+\infty$.

Remark 4.4. Unlike to Theorem 3.1, we are not able to compute explicitly the constant $c$ in in Theorem 4.3.

\section{Proof of the Neumann Results}

We begin this section by proving some auxiliary results that are essential to prove Theorem 4.3. The next lemma is a generalization for $p \geq 2$ of Oleinik's Lemma [26].

Lemma 5.1. Let $\Omega \subset \mathbb{R}^{N}$ be a bounded domain with $C^{1}$ boundary and, for $\delta>0$, let $G_{\delta}$ be a tubular neighborhood of $\partial \Omega$, i.e. $G_{\delta}=\{x \in \Omega$ : dist $(x, \partial \Omega)<\delta\}$. Then there exists $\delta_{0}>0$ such that for every $\delta \in\left(0, \delta_{0}\right)$ and every $v \in W^{1, p}(\Omega)$ we have

$$
\|v\|_{L^{p}\left(G_{\delta}\right)} \leq c \delta^{\frac{1}{p}}\|v\|_{W^{1, p}(\Omega)}
$$

where $c$ is a constant independent of $\delta$ and $v$.

Proof. Let $G_{\delta}=\{x \in \Omega: \operatorname{dist}(x, \partial \Omega)<\delta\}$, it follows that $S_{\delta}=\partial G_{\delta}$ are uniformly smooth surfaces.

By the Sobolev trace Theorem we have

$$
\|v\|_{L^{p}\left(S_{\delta}\right)}^{p}=\int_{S_{\delta}}|v|^{p} d S \leq c\|v\|_{W^{1, p}\left(\Omega_{\delta}\right)}^{p} \leq c\|v\|_{W^{1, p}(\Omega)}^{p} \quad \delta \in\left[0, \delta_{0}\right]
$$

where $c$ is a constant independent of $\delta$. Integrating this inequality with respect to $\delta$ we get

$$
\|v\|_{L^{p}\left(G_{\delta}\right)}^{p}=\int_{0}^{\delta}\left(\int_{S_{\tau}}|v|^{p} d S\right) d \tau \leq c \delta\|v\|_{W^{1, p}(\Omega)}^{p}
$$

and the Lemma is proved.

The next Theorem is essential to estimate the rate of convergence of the eigenvalues since it allows us to replace an integral involving a rapidly oscillating function with one that involves its average in the unit cube. First, we need an easy Lemma that computes the Poincaré constant on the cube of side $\varepsilon$ in terms of the Poincaré constant of the unit cube. Although this result is well known and its proof follows directly by a change of variables, we choose to include it for the sake of completeness.

Lemma 5.2. Let $Q$ be the unit cube in $\mathbb{R}^{N}$ and let $c_{p}$ be the Poincaré constant in the unit cube in $L^{p}$, i.e.

$$
\left\|u-(u)_{Q}\right\|_{L^{p}(Q)} \leq c_{p}\|\nabla u\|_{L^{p}(Q)}, \quad \text { for every } u \in W^{1, p}(Q),
$$


where $(u)_{Q}$ is the average of $u$ in $Q$. Then, for every $u \in W^{1, p}\left(Q_{\varepsilon}\right)$ we have

$$
\left\|u-(u)_{Q_{\varepsilon}}\right\|_{L^{p}\left(Q_{\varepsilon}\right)} \leq c_{p} \varepsilon\|\nabla u\|_{L^{p}\left(Q_{\varepsilon}\right)},
$$

where $Q_{\varepsilon}=\varepsilon Q$.

Proof. Let $u \in W^{1, p}\left(Q_{\varepsilon}\right)$. We can assume that $(u)_{Q_{\varepsilon}}=0$. Now, if we denote $u_{\varepsilon}(y)=u(\varepsilon y)$, we have that $u_{\varepsilon} \in W^{1, p}(Q)$ and by the change of variables formula, we get

$$
\int_{Q_{\varepsilon}}|u|^{p}=\int_{Q}\left|u_{\varepsilon}\right|^{p} \varepsilon^{n} \leq c_{p}^{p} \varepsilon^{n} \int_{Q}\left|\nabla u_{\varepsilon}\right|^{p}=c_{p}^{p} \varepsilon^{p} \int_{Q_{\varepsilon}}|\nabla u|^{p} .
$$

The proof is now complete.

Theorem 5.3. Let $\Omega \subset \mathbb{R}^{N}$ be a bounded domain with smooth boundary and denote by $Q$ the unit cube in $\mathbb{R}^{N}$. Let $g$ be a $Q$-periodic bounded function such that $(g)_{Q}=$ 0. Then the inequality

$$
\left|\int_{\Omega} g\left(\frac{x}{\varepsilon}\right) u v\right| \leq c \varepsilon\|u\|_{W^{1, p}(\Omega)}\|v\|_{W^{1, p^{\prime}}(\Omega)}
$$

holds for every $u \in W^{1, p}(\Omega)$ and $v \in W^{1, p^{\prime}}(\Omega)$, where $c$ is a constant independent of $\varepsilon, u, v$ and $p, p^{\prime}$ are conjugate exponents.

Proof. Denote by $I^{\varepsilon}$ the set of all $z \in \mathbb{Z}^{N}$ such that $Q_{z, \varepsilon}:=\varepsilon(z+Q) \subset \Omega$. Set $\Omega_{1}=\bigcup_{z \in I^{\varepsilon}} Q_{z, \varepsilon}$ and $G=\Omega \backslash \bar{\Omega}_{1}$. Let us consider the functions $\bar{v}$ and $\bar{u}$ given by the formulas

$$
\bar{v}(x)=\frac{1}{\varepsilon^{n}} \int_{Q_{z, \varepsilon}} v(x) d x, \quad \bar{u}(x)=\frac{1}{\varepsilon^{n}} \int_{Q_{z, \varepsilon}} u(x) d x
$$

for $x \in Q_{z, \varepsilon}$. Then we have

$$
\begin{aligned}
\int_{\Omega} g_{\varepsilon} u v & =\int_{G} g_{\varepsilon} u v+\int_{\Omega_{1}} g_{\varepsilon} u v \\
& =\int_{G} g_{\varepsilon} u v+\int_{\Omega_{1}} g_{\varepsilon}(u-\bar{u}) v+\int_{\Omega_{1}} g_{\varepsilon} \bar{u}(v-\bar{v})+\int_{\Omega_{1}} g_{\varepsilon} \bar{v} \bar{u} .
\end{aligned}
$$

The set $G$ is a $\delta$-neighborhood of $\partial \Omega$ with $\delta=c \varepsilon$ for some constant $c$, and therefore according to Lemma 5.1 we have

$$
\begin{gathered}
\|u\|_{L^{p}(G)} \leq c \varepsilon^{\frac{1}{p}}\|u\|_{W^{1, p}(\Omega)} \\
\|v\|_{L^{p^{\prime}}(G)} \leq c \varepsilon^{\frac{1}{p^{\prime}}}\|v\|_{W^{1, p^{\prime}}(\Omega)} .
\end{gathered}
$$

As $g$ is bounded, we get

$$
\int_{G} g_{\varepsilon} u v \leq c\|u\|_{L^{p}(G)}\|v\|_{L^{p^{\prime}}(G)} \leq c \varepsilon\|u\|_{W^{1, p}(\Omega)}\|v\|_{W^{1, p^{\prime}}(\Omega)} .
$$

Now, by Lema 5.2 we get

$$
\begin{aligned}
\|u-\bar{u}\|_{L^{p}\left(\Omega_{1}\right)} & =\left(\sum_{z \in I^{\varepsilon}} \int_{Q_{z, \varepsilon}}|u-\bar{u}|^{p} d x\right)^{\frac{1}{p}} \leq c_{p} \varepsilon\left(\sum_{z \in I_{z, \varepsilon}} \int_{Q_{z, \varepsilon}}|\nabla u(x)|^{p} d x\right)^{\frac{1}{p}} \\
& =c_{p} \varepsilon\|\nabla u\|_{L^{p}\left(\Omega_{1}\right)} .
\end{aligned}
$$


Analogously

$$
\|v-\bar{v}\|_{L^{p^{\prime}}\left(\Omega_{1}\right)} \leq c_{p^{\prime}} \varepsilon\|\nabla v\|_{L^{p^{\prime}}\left(\Omega_{1}\right)}
$$

By the definition of $\bar{u}(x)$ we get

$$
\begin{aligned}
\|\bar{u}\|_{L^{p}\left(\Omega_{1}\right)}^{p} & =\sum_{z \in I^{\varepsilon}} \int_{Q_{z, \varepsilon}}|\bar{u}|^{p}=\sum_{z \in I^{\varepsilon}} \varepsilon^{n}\left(\varepsilon^{-n} \int_{Q_{z, \varepsilon}} u\right)^{p} \\
& \leq \varepsilon^{n-n p} \sum_{z \in I^{\varepsilon}}\left|Q_{z, \varepsilon}\right|^{p / p^{\prime}} \int_{Q_{z, \varepsilon}}|u|^{p}=\varepsilon^{n-n p+n p / p^{\prime}} \sum_{z \in I^{\varepsilon}} \int_{Q_{z, \varepsilon}}|u|^{p} \\
& =\int_{\Omega_{1}}|u|^{p}=\|u\|_{L^{p}\left(\Omega_{1}\right)}^{p} .
\end{aligned}
$$

Finally, since $(g)_{Q_{1}}=0$ and since $g$ is $Q$-periodic, we get

$$
\int_{\Omega_{1}} g_{\varepsilon} \bar{u} \bar{v}=\sum_{z \in I^{\varepsilon}} \bar{u} \bar{v} \int_{Q_{z, \varepsilon}} g_{\varepsilon}=0 .
$$

Now, combining (5.3), (5.4), (5.5), (5.6) and (5.7) we can bound (5.1) by

$$
\int_{\Omega} g_{\varepsilon} u v \leq C \varepsilon\|u\|_{W^{1, p}(\Omega)}\|v\|_{W^{1, p^{\prime}}(\Omega)}
$$

This finishes the proof.

Now we are ready to proof Theorem 4.3:

Proof of Theorem 4.3: The result follows applying Theorem 5.3 to $\widetilde{g}_{\epsilon}=g_{\epsilon}-\bar{g}$ and taking $v \equiv 1$.

Remark 5.4. Let us observe that $u \in W^{1, p}(\Omega)$ is solution of equation (4.1) if and only if $u$ is solution of equation

$$
-\Delta_{p} u+m\left(u^{+}\right)^{p-1}+n\left(u^{-}\right)^{p-1}=\tilde{\alpha} m\left(u^{+}\right)^{p-1}-\tilde{\beta} n\left(u^{-}\right)^{p-1} \quad \text { in } \Omega .
$$

with Neumann boundary conditions, where $\tilde{\alpha}=\alpha-1$ and $\tilde{\beta}=\beta+1$. The main advantage between consider equations (4.1) and (5.8) is the fact that in the second one the functional $A(u)$ defined in (1.7) becomes in

$$
A_{m, n}(u)=\int_{\Omega}|\nabla u|^{p}+m\left(u^{+}\right)^{p}+n\left(u^{-}\right)^{p} d x,
$$

which involves both $\nabla u$ and the function $u$.

Proof of Theorem 4.2: The proof is similar to that of Theorem 2.6 for the Dirichlet case. According to Remark 5.4 we consider equation $(5.8)$. Let $\left(\tilde{\alpha}_{\varepsilon}, \tilde{\beta}_{\varepsilon}\right)$ be a point belonging to the curve $\mathcal{C}_{1}^{\varepsilon}\left(m_{\varepsilon}, n_{\varepsilon}\right)$ and let $\left(\tilde{\alpha}_{0}, \tilde{\beta}_{0}\right)$ be the point obtained when $\varepsilon \rightarrow 0$. It follows that $\left(\tilde{\alpha}_{0}, \tilde{\beta}_{0}\right)$ belongs to the spectrum of the limit equation. Let us see that it belongs to $\mathcal{C}(\bar{m}, \bar{n})$. The main difference is that in the characterization (4.4) of $c\left(m_{\varepsilon}, n_{\varepsilon}\right)$, now we are considering

$$
\Gamma=\left\{\gamma \in C\left(J, W^{1, p}(\Omega)\right): \gamma(0) \geq 0 \text { and } \gamma(1) \leq 0\right\} .
$$


with $J:=[0,1]$. Fixed a value of $\varepsilon>0$ we write

$$
c\left(m_{\varepsilon}, n_{\varepsilon}\right)=\inf _{\gamma \in \Gamma} \sup _{u \in \gamma} \frac{A_{m_{\varepsilon}, n_{\varepsilon}}(u)}{B_{m_{\varepsilon}, n_{\varepsilon}}(u)} .
$$

By (1.5) and (5.10) we have the following characterization of $\tilde{\alpha}_{\varepsilon}(s)$

$$
\tilde{\alpha}_{\varepsilon}(s)=c\left(m_{\varepsilon}, s n_{\varepsilon}\right)=\inf _{\gamma \in \Gamma} \sup _{u \in \gamma} \frac{A_{m_{\varepsilon}, n_{\varepsilon}}(u)}{B_{m_{\varepsilon}, s n_{\varepsilon}}(u)} .
$$

Similarly, we can consider an equation analog to (5.11) for the representation of $\tilde{\alpha}_{0}(s)$. Let $\delta>0$ and $\gamma_{1}=\gamma_{1}(\delta) \in \Gamma$ such that

$$
\tilde{\alpha}_{0}(s)=\sup _{u \in \gamma_{1}} \frac{A_{\bar{m}, \bar{n}}(u)}{B_{\bar{m}, s \bar{n}}(u)}+O(\delta) .
$$

In order to find a bound for $\tilde{a}_{\varepsilon}$ we use $\gamma_{1} \in \Gamma$, which is admissible in its variational characterization,

$$
\tilde{\alpha}_{\varepsilon}(s) \leq \sup _{u \in \gamma_{1}} \frac{A_{m_{\varepsilon}, s n_{\varepsilon}}(u)}{B_{\bar{m}, s \bar{n}}(u)} \frac{B_{\bar{m}, s \bar{n}}(u)}{B_{m_{\varepsilon}, s n_{\varepsilon}}(u)}
$$

To bound $\tilde{\alpha}_{\varepsilon}$ we look for bounds of the two quotients in (5.13). Since $u \in W^{1, p}(\Omega)$, by Theorem 4.3 we obtain that

$$
\frac{A_{m_{\varepsilon}, n_{\varepsilon}}(u)}{B_{\bar{m}, s \bar{n}}(u)} \leq \frac{A_{\bar{m}, \bar{n}}(u)}{B_{\bar{m}, s \bar{n}}(u)}+\frac{c \varepsilon\left\|\left|u^{+}\right| p\right\|_{W^{1,1}(\Omega)}+c \varepsilon\left\|\left|u^{-}\right|^{p}\right\|_{W^{1,1}(\Omega)}}{B_{\bar{m}, s \bar{n}}(u)} .
$$

For every function $u \in \gamma_{1}$ we have that

$$
\frac{A_{\bar{m}, \bar{n}}(u)}{B_{\bar{m}, s \bar{n}}(u)} \leq \sup _{u \in \gamma_{1}} \frac{A_{\bar{m}, \bar{n}}(u)}{B_{\bar{m}, s \bar{n}}(u)}=\tilde{\alpha}_{0}(s)+O(\delta) .
$$

By using Young inequality, for each $v \in W^{1, p}(\Omega)$

$$
\begin{aligned}
\left\||v|^{p}\right\|_{W^{1,1}(\Omega)} & =\left\||v|^{p}\right\|_{L^{1}(\Omega)}+p\left\||v|^{p-1} \nabla v\right\|_{L^{1}(\Omega)} \\
& =\|v\|_{L^{p}(\Omega)}^{p}+p\left\||v|^{p-1} \nabla v\right\|_{L^{1}(\Omega)} \\
& \leq p\|v\|_{L^{p}(\Omega)}^{p}+\|\nabla v\|_{L^{p}(\Omega)}^{p} .
\end{aligned}
$$

From (5.15) it follows that

$$
\begin{aligned}
\frac{\left\|u^{+} \mid{ }^{p}\right\|_{W^{1,1}(\Omega)}}{B_{\bar{m}, s \bar{n}}(u)} & \leq \frac{p\left\|u^{+}\right\|_{L^{p}(\Omega)}^{p}+\left\|\nabla u^{+}\right\|_{L^{p}(\Omega)}^{p}}{B_{\bar{m}, s \bar{n}}(u)} \\
& \leq c \frac{A_{\bar{m}, \bar{n}}(u)}{B_{\bar{m}, s \bar{n}}(u)} \\
& \leq c \sup _{u \in \gamma_{1}} \frac{A_{\bar{m}, \bar{n}}(u)}{B_{\bar{m}, s \bar{n}}(u)} \\
& =c\left(\tilde{\alpha}_{0}(s)+O(\delta)\right)
\end{aligned}
$$

and similarly

$$
\frac{\left\|\left|u^{-}\right|^{p}\right\|_{W^{1,1}(\Omega)}}{B_{\bar{m}, s \bar{n}}(u)} \leq c\left(\tilde{\alpha}_{0}(s)+O(\delta)\right) .
$$


To bound the second quotient in (5.13), we use again Theorem 4.3 and (2.1) to obtain

$$
\begin{aligned}
\frac{\int_{\Omega} \bar{m}\left|u^{+}\right|^{p}}{B_{m_{\varepsilon}, s n_{\varepsilon}}(u)} & \leq \frac{\int_{\Omega} m_{\varepsilon}\left|u^{+}\right|^{p}}{B_{m_{\varepsilon}, s n_{\varepsilon}}(u)}+c \varepsilon \frac{\left\|\left|u^{+}\right|^{p}\right\|_{W^{1,1}(\Omega)}}{B_{m_{\varepsilon}, s n_{\varepsilon}}(u)} \\
& \leq \frac{\int_{\Omega} m_{\varepsilon}\left|u^{+}\right|^{p}}{B_{m_{\varepsilon}, s n_{\varepsilon}}(u)}+c \varepsilon \frac{\left\|\left|u^{+}\right|^{p}\right\|_{W^{1,1}(\Omega)}}{B_{\bar{m}, s \bar{n}}(u)}
\end{aligned}
$$

and similarly

$$
\frac{\int_{\Omega} s \bar{n}\left|u^{-}\right|^{p}}{B_{m_{\varepsilon}, s n_{\varepsilon}}(u)} \leq \frac{\int_{\Omega} s n_{\varepsilon}\left|u^{+}\right|^{p}}{B_{m_{\varepsilon}, s n_{\varepsilon}}(u)}+s c \varepsilon \frac{\left\|\left|u^{-}\right|^{p}\right\|_{W^{1,1}(\Omega)}}{B_{\bar{m}, s \bar{n}}(u)} .
$$

Now, from equations (5.18),(5.19) together with (5.16) and (5.17) we get

$$
\begin{aligned}
\frac{B_{\bar{m}, s \bar{n}}(u)}{B_{m_{\varepsilon}, s n_{\varepsilon}}(u)} & =\frac{\int_{\Omega} \bar{m}\left|u^{+}\right|^{p}+\int_{\Omega} s \bar{n}\left|u^{-}\right|^{p}}{B_{m_{\varepsilon}, s n_{\varepsilon}}(u)} \\
& \leq 1+(1+s) c \varepsilon\left(\tilde{\alpha}_{0}(s)+O(\delta)\right) .
\end{aligned}
$$

Then combining (5.13),(5.16),(5.17) and (5.20) we find that

$$
\tilde{\alpha}_{\varepsilon}(s) \leq\left(\left(\tilde{\alpha}_{0}(s)+O(\delta)\right)+c \varepsilon\left(\tilde{\alpha}_{0}(s)+O(\delta)\right)\right)\left(1+(1+s) c \varepsilon\left(\tilde{\alpha}_{0}(s)+O(\delta)\right)\right) .
$$

Letting $\delta \rightarrow 0$ we get

$$
\tilde{\alpha}_{\varepsilon}(s)-\tilde{\alpha}_{0}(s) \leq c \varepsilon\left(\tilde{\alpha}_{0}^{2}(1+s)+\tilde{\alpha}_{0}\right) .
$$

In a similar way, interchanging the roles of $\tilde{\alpha}_{0}$ and $\tilde{\alpha}_{\varepsilon}$, we obtain

$$
\tilde{\alpha}_{0}(s)-\tilde{\alpha}_{\varepsilon}(s) \leq c \varepsilon\left(\tilde{\alpha}_{\varepsilon}^{2}(1+s)+\tilde{\alpha}_{\varepsilon}\right) .
$$

From (5.21) and (5.22) we arrive at

$$
\left|\tilde{\alpha}_{0}(s)-\tilde{\alpha}_{\varepsilon}(s)\right| \leq c \varepsilon(1+s) \max \left\{\tilde{\alpha}_{0}(s)^{2}, \tilde{\alpha}_{\varepsilon}(s)^{2}\right\} .
$$

Now, using Lemma 3.3,

$$
\left|\alpha_{\varepsilon}(s)-\alpha_{0}(s)\right| \leq c(1+s) \tau(s)^{2} \varepsilon,
$$

where $c$ is a constant independent of $\varepsilon$ and $s$, and $\tau(s)$ is given by (3.8). Here, Lemma 3.3 holds in the Neumann case, but now we have

$$
\alpha(s) \leq \min \left\{m_{-}^{-1}, n_{-}^{-1}\right\} \mu_{2} \tau(s), \quad \beta(s) \leq \min \left\{m_{-}^{-1}, n_{-}^{-1}\right\} \mu_{2} s \tau(s)
$$

with $\mu_{2}$ the second eigenvalue of the $p$-Laplacian equation on $\Omega$ with Neumann boundary conditions. From the convergence of $\alpha_{\varepsilon}$ and (1.5) it follows the convergence of $\beta_{\varepsilon}$ and of the whole curve.

Proof of Theorem 4.1: As Theorem 3.6 holds for functions belonging to $W^{1, p}(\Omega)$ with $\Omega$ any bounded domain in $\mathbb{R}^{N}$, this proof is analogous to those of Theorem 2.1 .

\section{ACKNOWLEDGEMENTS}

I want to thank Julián Fernández Bonder for valuable help and many stimulating discussions on the subject of the paper. 


\section{REFERENCES}

1. M. Alif and J.-P. Gossez, On the Fučík spectrum with indefinite weights, Differential Integral Equations 14 (2001), no. 12, 1511-1530. MR 1859919 (2002f:34024) 2

2. A. Anane, O. Chakrone, and M. Moussa, Spectrum of one dimensional p-Laplacian operator with indefinite weight, Electron. J. Qual. Theory Differ. Equ. (2002), No. 17, 11. MR 1942086 (2004b:34044) 1

3. M. Arias and J. Campos, Fučik spectrum of a singular Sturm-Liouville problem, Nonlinear Anal. 27 (1996), no. 6, 679-697. MR 1399068 (97g:34029) 2

4. M. Arias, J. Campos, M. Cuesta, and J.-P. Gossez, Asymmetric elliptic problems with indefinite weights, Ann. Inst. H. Poincaré Anal. Non Linéaire 19 (2002), no. 5, 581-616. MR 1922470 (2003g:35169) 2, 4, 5, 8

5. _ An asymmetric Neumann problem with weights, Ann. Inst. H. Poincaré Anal. Non Linéaire 25 (2008), no. 2, 267-280. MR 2396522 (2009b:35137) 10

6. L. Baffico, C. Conca, and M. Rajesh, Homogenization of a class of nonlinear eigenvalue problems, Proc. Roy. Soc. Edinburgh Sect. A 136 (2006), no. 1, 7-22. MR 2217505 (2007b:35021) 3,4

7. Andrea Braides, Valeria Chiadò Piat, and Anneliese Defranceschi, Homogenization of almost periodic monotone operators, Ann. Inst. H. Poincaré Anal. Non Linéaire 9 (1992), no. 4, 399-432. MR 1186684 (94d:35014) 3

8. C. Castro and E. Zuazua, High frequency asymptotic analysis of a string with rapidly oscillating density, European J. Appl. Math. 11 (2000), no. 6, 595-622. MR 1811309 (2001k:34093) 3

9. Carlos Castro and Enrique Zuazua, Low frequency asymptotic analysis of a string with rapidly oscillating density, SIAM J. Appl. Math. 60 (2000), no. 4, 1205-1233 (electronic). MR 1760033 (2001h:34117) 3

10. M. Cuesta, D. de Figueiredo, and J.-P. Gossez, The beginning of the Fučik spectrum for the p-Laplacian, J. Differential Equations 159 (1999), no. 1, 212-238. MR 1726923 (2001f:35308) 1,2

11. E. N. Dancer, On the Dirichlet problem for weakly non-linear elliptic partial differential equations, Proc. Roy. Soc. Edinburgh Sect. A 76 (1976/77), no. 4, 283-300. MR 0499709 (58 \#17506) 2

12. Norman Dancer and Kanishka Perera, Some remarks on the Fučik spectrum of the pLaplacian and critical groups, J. Math. Anal. Appl. 254 (2001), no. 1, 164-177. MR 1807894 (2001k:35238) 2

13. A. Derlet, J.-P. Gossez, and P. Takáč, Minimization of eigenvalues for a quasilinear elliptic Neumann problem with indefinite weight, J. Math. Anal. Appl. 371 (2010), no. 1, 69-79. MR 2660987 (2011h:49069) 1

14. P. Drábek, Solvability and bifurcations of nonlinear equations, Pitman Research Notes in Mathematics Series, vol. 264, Longman Scientific \& Technical, Harlow, 1992. MR 1175397 (94e:47084) 2

15. Pavel Drábek and Stephen B. Robinson, On the generalization of the Courant nodal domain theorem, J. Differential Equations 181 (2002), no. 1, 58-71. MR 1900460 (2003g:35170) 2

16. Siham El Habib and Najib Tsouli, On the spectrum of the p-Laplacian operator for Neumann eigenvalue problems with weights, Proceedings of the 2005 Oujda International Conference on Nonlinear Analysis (San Marcos, TX), Electron. J. Differ. Equ. Conf., vol. 14, Southwest Texas State Univ., 2006, pp. 181-190 (electronic). MR 2316116 (2008b:35079) 1

17. Svatopluk Fučík, Boundary value problems with jumping nonlinearities, Časopis Pěst. Mat. 101 (1976), no. 1, 69-87. MR 0447688 (56 \#5998) 2

18. Svatopluk Fučík and Alois Kufner, Nonlinear differential equations, Studies in Applied Mechanics, vol. 2, Elsevier Scientific Publishing Co., Amsterdam, 1980. MR 558764 (81e:35001) 2

19. T. Godoy, J.-P. Gossez, and S. Paczka, On the antimaximum principle for the p-Laplacian with indefinite weight, Nonlinear Anal. 51 (2002), no. 3, 449-467. MR 1942756 (2003i:35221) 10

20. Julián Fernández Bonder, Juan P. Pinasco and Ariel M. Salort, Eigenvalue homogenization for quasilinear elliptic equations with different boundary conditions, preprint, arXiv:1208.5744v1 (2012). 3 
21. Convergence rate for quasilinear eigenvalue homogenization, preprint, arXiv:1211.0182v1 (2012). 3, 4, 5, 6

22. Carlos E. Kenig, Fanghua Lin, and Zhongwei Shen, Convergence rates in $l^{2}$ for elliptic homogenization problems, to appear in archive for rational mechanics and analysis, arXiv:1103.0023v1 (2011). 3

23. Liamidi Leadi and Aboubacar Marcos, On the first curve in the Fučik spectrum with weights for a mixed p-Laplacian, Int. J. Math. Math. Sci. (2007), Art. ID 57607, 13. MR 2365740 (2008h:35094) 2

24. R. Lemmert and W. Walter, Singular nonlinear boundary value problems, Appl. Anal. 72 (1999), no. 1-2, 191-203. MR 1775441 (2001d:34040) 2

25. Vladimir G. Maz'ja, Sobolev spaces, Springer Series in Soviet Mathematics, Springer-Verlag, Berlin, 1985, Translated from the Russian by T. O. Shaposhnikova. MR 817985 (87g:46056)

26. O. A. Oleĭnik, A. S. Shamaev, and G. A. Yosifian, Mathematical problems in elasticity and homogenization, Studies in Mathematics and its Applications, vol. 26, North-Holland Publishing Co., Amsterdam, 1992. MR 1195131 (93k:35025) 3, 12

27. Kanishka Perera, On the Fučik spectrum of the p-Laplacian, NoDEA Nonlinear Differential Equations Appl. 11 (2004), no. 2, 259-270. MR 2210289 (2006m:35270) 2

28. Bryan P. Rynne, The Fučik spectrum of general Sturm-Liouville problems, J. Differential Equations 161 (2000), no. 1, 87-109. MR 1740358 (2000j:34031) 2

Departamento de Matemática

FCEN - Universidad de Buenos Aires and

IMAS - CONICET.

Ciudad Universitaria, Pabellón I

(1428) Av. Cantilo s/N.

Buenos Aires, Argentina.

E-mail address: asalort@dm.uba.ar 